\title{
Spectrum sensing with spatial signatures in the presence of noise uncertainty and shadowing
}

\author{
Sadiq Ali, Gonzalo Seco-Granados and José A López-Salcedo
}

\begin{abstract}
In this paper, we consider a system of cognitive radios that collaborate with each other with the aim of detecting the random waveforms being emitted from licensed users. We study the problem of fusing the statistics from collaborating sensors, assuming that they send their statistics to a base station, where the final decision is made. The main contribution of this work is the derivation of a cognitive detector based on the generalized likelihood ratio test and the use of spatial signatures, a novel concept that allows the detector to capture the spatial correlation inherently embedded in measurements coming from neighboring sensors. The problem is formulated in terms of a model order detection problem, where a set of active and inactive sensors can be distinguished, thus allowing the detector to operate with a rank-reduced version of the observed covariance matrix. Since the estimation of this matrix may be a challenge in large-scale networks, we study the application of shrinkage techniques to cope with the problem of having more sensors than available observations. Finally, we analyze the performance of the proposed detection scheme in the presence of log-normal shadowing effects and noise power uncertainties, the latter due to presence of interferences. For the proposed detector, numerical results are drawn, showing a significant gain in performance compared to traditional approaches.
\end{abstract}

\section{Introduction}

Due to the rapid growth in the field of radio communication, most of the available spectrum has already become congested, and the assignment of frequencies to new services is currently a critical problem. Nevertheless, studies show that assigned frequencies are not occupied all the time, implying that the traditional way of spectrum allocation has resulted in underutilization of such a precious resource. In that sense, cognitive radio (CR) has the potential to become the solution to the spectrum underutilization problem. The CR paradigm is based upon the coexistence within the same frequency band, of both licensed and unlicensed users, in such a way that the latter are allowed to utilize the free spectrum holes left by the former in a dynamic and opportunistic manner [1,2]. This technology, which is currently on the forefront of next-generation wireless systems, and regulatory as well as standardization bodies are starting to support the idea of spectrum reuse $[3,4]$. Among

\footnotetext{
*Correspondence: sadiq.ali@uab.es

Department of Telecommunications and Systems Engineering, Engineering School, Universitat Autònoma de Barcelona (UAB), Bellaterra, Barcelona 08193, Spain
}

the various functions of a CR system, reliable sensing of the licensed or primary users' (PU) spectrum is certainly of paramount importance. Such spectrum sensing is performed by unlicensed or secondary users (SU), either following a single-sensor or a multisensor approach. The process of spectrum sensing with a single sensor is fundamentally limited by local impairments, such as the noise level, the signal-to-noise ratio (SNR) wall [5], and radio propagation effects such as path loss and fading experienced by this sensor, which significantly deteriorate its sensing performance [6]. In contrast, collaborative spectrum sensing relies on the combination of measurements coming from multiple neighboring sensors [7]. Therefore, collaborative approaches are able to circumvent most of the propagation impairments of single-sensor spectrum sensing due to the presence of diversity in the set of measurements being processed at the fusion center [8]. It has to be taken into account that for the case of large-scale sensor networks, the signal of the PU will only reach to a subset of sensors (i.e., those sensors located close to the PU), which will typically be closely spaced, thus forming a cluster having highly correlated observations [9]. This observation motivates our interest on exploiting the

\section{是 Springer}

(c) 2013 Ali et al: licensee Springer. This is an Open Access article distributed under the terms of the Creative Commons Attribution License (http://creativecommons.org/licenses/by/2.0), which permits unrestricted use, distribution, and reproduction in any medium, provided the original work is properly cited. 
spatial information of the received signal at closely spaced sensors, with the aim of providing an additional degree of robustness to the overall network decision metric.

There have been some attempts to consider correlated measurements into the formulation of collaborative signal detection. However, many of these studies consider the presence of correlation as a deleterious effect $[10,11]$ rather than as a form of side information that can be used to enhance the detection performance. Similarly, most of the work done on correlated detection problems just focuses on the discrimination between correlated and independent observations, by means of exploiting the structure of the covariance matrix. For this particular problem, the study in [12, Chap. 9-10] extensively discusses the use of multivariate detectors for testing the independence of random observations with the help of the generalized likelihood ratio test (GLRT) based on covariance matrices. These GLRT-based detectors typically end up with a simple quotient between the determinant of the sample covariance matrix and the determinant of its diagonal version. Recently, covariance-based detection techniques in [12] have been widely adopted for the detection of signals with distributed sensors, especially in the context of cognitive radios $[13,14]$. However, these detectors typically focus on detecting the presence of correlated data, as a possible indication of the presence of signal from a PU. They do not focus, instead, on exploiting the actual correlation structure that impinges onto the sensor field when an emitting PU is present. This observation suggests that the performance can be further improved by exploiting the sensor proximity information, leading to new schemes based on the concept of location awareness [15-17]. For the case of collaborative spectrum sensing, prior work has demonstrated that information on the sensor position can lead to more reliable spectrum sensing, thus confirming the convenience of this information, when available $[10,18]$.

Motivated by these facts, we propose a modified GLRTbased detector that achieves the regularization of the unknown covariance matrix with the help of spatial signatures. The concept of signatures would be somehow equivalent to steering vectors in the field of array signal processing [19], which are adopted herein as a way to capture the structure of spatially correlated measurements between neighboring sensors. Furthermore, selecting just some of the sensors of the network allows the proposed detector to operate on a rank-reduced subspace of the received signal, thus achieving a significant SNR gain. This approach, which was preliminary introduced in our earlier work [20], is extended herein to the problem of detecting Gaussian random waveforms emitted from a PU with unknown covariance matrix. This is in contrast to the deterministic approach considered in [20] where an unknown but constant waveform was assumed to be transmitted by the PU and where ideal propagation conditions as well as perfect knowledge on the signal parameters was also assumed. In that sense, the present contribution offers a much more realistic approach by assuming the emission of random waveforms, by including the presence of shadowing and noise power uncertainty, and by taking into account the practical problems that may arise in large-scale networks when estimating the unknown covariance matrix of the PU. The latter is indeed related to the required number of observations to avoid ill-conditioning in the estimation of this matrix, which is typically on the order of the number of sensors [21]. Therefore, detection algorithms requiring the inverse or the determinant of this matrix can no longer be applied for short observation periods. To cope with this practical problem, the present work incorporates the concept of shrinkage estimation, a method that is found to improve the stability of estimated covariance matrices with short data records [21]. Simulation results have been obtained to compare the proposed detection schemes with and without spatial signatures, as well as with and without shrinkage estimation, showing that the introduction of spatial structures and shrinkage estimation significantly improves the overall detection performance.

The remaining of the paper is organized as follows. In Section 2, the problem statement and details about the signal model are presented. Section 3 presents the structured signal model based on the concept of spatial signatures, and 4 introduces the proposed detection algorithm. In 5 we briefly discuss the shrinkage method for estimating the covariance matrix. Finally, simulation results are presented in 6, and conclusions are drawn in Section 7.

\section{Problem statement}

We consider herein a large cognitive radio network where both primary and secondary users coexist in the same geographical area. We assume an infrastructure-based secondary network [22], where each cell consists of a single base station (BS) working as a fusion center and $K$ SUs working as sensors. We also assume that sensors are deployed in the region following a uniform distribution and that the sensors and PU remain stationary in their position during the observation interval. The signal power emitted by the PU decays isotropically as a function of distance and is affected by a significant path loss attenuation due to the large area being covered by the network, as well as by fading/shadowing effects. As a consequence, only a subset of sensors will be able to receive enough power levels so as to easily detect the presence of the PU with a given detection performance $[23,24]$. The rest of the sensors will typically receive extremely weak power levels, and this observation allows us to distinguish between the so-called active and inactive sensors, respectively. In the process of collaborative spectrum sensing, the BS 
coordinates the opportunistic spectrum access of all SUs within its cell. This is done by directing sensors to perform spectrum sensing periodically. At the end of each sensing period, all the sensors report their measurements to the BS, which makes the final decision about the presence or absence of the PU [8]. Once the final decision is made at the BS, it is broadcast back to SUs within the cell in order to inform them about the presence or an absence of the PU. Similarly to [25], we further assume that the BS knows the location of the SUs, either through the use of positioning techniques or through some calibration process.

\subsection{Signal model and test statistics at the SU}

In the collaborative sensing system considered herein, we assume that sensors simply measure the PU signal power on a target frequency band using an energy detector, and they report their sensing results to the BS [26]. This is a simplistic interpretation of collaborative sensing, which indeed covers a much wider area [27], but it allows us to concentrate on the specific problem of energy detection. Indeed, the energy detector is the simplest detector that can be constructed in practice. It uses very limited a priori information regarding the signal since the detection is based only on the received signal power. In the sequel, we will consider an observation interval of $n=1,2, \ldots, N$ sensing periods. During the $n$th sensing period, every SU captures a snapshot of $m=1,2, \ldots, M$ received signal samples in order to estimate the received signal power. At the $i$ th sensor (i.e., $\mathrm{SU}$ ), the corresponding received samples are denoted by $y_{i}(m ; n)$, and two possible hypotheses arise for the spectrum sensing problem under study. On the one hand, we have the null hypothesis denoted by $\mathcal{H}_{0}$, which represents the case in which no PU signal is present in the received samples $y_{i}(m ; n)$. On the other hand, we have the signal-present hypothesis denoted by $\mathcal{H}_{1}$ which represents the case in which some PU signal is actually present in these samples. The signal model for these two hypotheses can be formulated as follows:

$$
\begin{aligned}
& \mathcal{H}_{0}: y_{i}(m ; n)=z_{i}(m ; n) \\
& \mathcal{H}_{1}: y_{i}(m ; n)=g_{i}(m ; n)+z_{i}(m ; n)
\end{aligned} i=1,2, \ldots, K,
$$

where $z_{i}(m ; n)$ are the i.i.d. zero-mean samples encompassing the aggregate of random disturbances affecting each sensor, whereas $g_{i}(m ; n)$ are the received signal samples corresponding to the random waveform emitted from the PU. Based on these samples, the energy detector at sensor $i$ for the $n$th sensing period is given by:

$$
T_{i}(n)=\frac{1}{M} \mathbf{y}_{i}^{T}(n) \mathbf{y}_{i}(n)=\frac{1}{M} \sum_{m=1}^{M} y_{i}^{2}(m ; n)
$$$$
\text { where } \mathbf{y}_{i}(n) \triangleq\left[y_{i}(1 ; n), y_{i}(2 ; n), \ldots, y_{i}(M ; n)\right]^{T} \text {. }
$$

It is interesting to note that the energy detector in (2) can fairly be approximated by a Gaussian distribution in virtue of the central limit theorem (CLT), provided that $M$ is sufficiently large ${ }^{1}$. Moreover, it should also be taken into account that the overall noise in a wireless receiver is often considered to be an ensemble of various effects, including not only the thermal noise contribution but also other degradations such as the presence of interference signals from distant PUs or from other opportunistic SUs. All these random disturbances are included within the $z_{i}(m ; n)$ samples in $(1)$, whose overall unknown power will be denoted herein by $\sigma_{\varepsilon, i}^{2} \doteq \mathrm{E}\left[\left|z_{i}(m ; n)\right|^{2}\right]$. For the sake of clarity, we will loosely refer to these samples as noise samples. In practice, and because of the unknown and random nature of the underlying disturbances, it is very difficult to determine the exact noise powers $\sigma_{\varepsilon, i}^{2}$ even if we calibrate the system [28]. In some situations, this noise power uncertainty may lead to an increase of the SNR wall, which can be understood as the minimum SNR below which a signal cannot be detected, thus hindering the overall detection process [5]. Consequently, and from a practical point of view, it is of interest to assume that $\sigma_{\epsilon, i}^{2}$ is unknown. A similar statement can be made for the power being received from the PU at sensor $i$, which is referred herein as $P_{\varepsilon, i} \triangleq \mathrm{E}\left[\left|g_{i}(m ; n)\right|^{2}\right]$, and is also considered to be unknown due to the unknown location of the PU and the presence of shadowing/fading that may alter the actual received power from its nominal value.

With the above considerations, and in virtue of the Gaussian assumption in (2) provided by the CLT, the test statistics for the energy detector at sensor $i$ can be modeled by the following Gaussian distribution [26]:

$$
\begin{aligned}
& \mathcal{H}_{0}: T_{i}(n) \sim \mathcal{N}\left(\sigma_{\varepsilon, i}^{2}, \frac{2 \sigma_{\varepsilon, i}^{4}}{M}\right) \\
& \mathcal{H}_{1}: T_{i}(n) \sim \mathcal{N}\left(P_{\varepsilon, i}+\sigma_{\varepsilon, i}^{2}, \frac{2\left(P_{\varepsilon, i}^{2}+2 P_{\varepsilon, i} \sigma_{\varepsilon, i}^{2}\right)}{M}+\frac{2 \sigma_{\varepsilon, i}^{4}}{M}\right)
\end{aligned}
$$

where both $\sigma_{\varepsilon, i}^{2}$ and $P_{\varepsilon, i}$ are assumed to remain constant during the whole observation interval of $N$ sensing periods, and thus, they can be treated as unknown deterministic parameters herein. For the sake of clarity, note that we have used the notation $\mathcal{N}\left(\mu, \sigma^{2}\right)$ for some $\left\{\mu, \sigma^{2}\right\}$ in order to represent a Gaussian (i.e., normal) distribution with mean $\mu$ and variance $\sigma^{2}$.

\subsection{Signal model and test statistics at the BS}

Every sensor calculates an estimate of its received power level according to (2) and transmits this power estimate to the BS through a reporting channel. At the BS, the power estimates received from the $K$ sensors at the $n$th sensing period are stacked into the $(K \times 1)$ vector $\mathbf{x}(n) \triangleq$ 
$\left[x_{1}(n), \ldots, x_{K}(n)\right]^{T}$, where $x_{i}(n)$ stands for the noisy and attenuated version of $T_{i}(n)$ after propagation through the reporting channel from sensor $i$ to the BS [29]. Although the actual propagation effects of the $K$ reporting channels are assumed to be unknown herein, they are considered to remain constant within the observation interval of $N$ sensing periods. Therefore, the received signal model at the BS can still be expressed as some noisy contribution under hypothesis $\mathcal{H}_{0}$ and some signal plus noise contribution under hypothesis $\mathcal{H}_{1}$ as follows:

$$
\begin{aligned}
& \mathcal{H}_{0}: \mathbf{x}(n)=\mathbf{w}(n) \\
& \mathcal{H}_{1}: \mathbf{x}(n)=\mathbf{s}(n)+\mathbf{w}(n)
\end{aligned}
$$

for $n=1, \ldots, N$ and with $\mathbf{w}(n) \sim \mathcal{N}\left(\mu_{\mathbf{w}}, \Sigma_{\mathbf{w}}\right)$ a $(K \times 1)$ vector containing the reported noise power levels at each sensor when no PU is present, whereas $\mathbf{s}(n) \sim \mathcal{N}\left(\mu_{\mathbf{s}}, \Sigma_{\mathbf{s}}\right)$ is a $(K \times 1)$ vector with the PU power levels. It is important to recall here that both $\mathbf{w}(n)$ and $\mathbf{s}(n)$ are power measurements, and thus, the mean vectors $\mu_{\mathbf{w}} \triangleq \mathrm{E}[\mathbf{w}(n)]$ and $\mu_{\mathbf{s}} \triangleq \mathrm{E}[\mathbf{s}(n)]$ contain the mean noise powers and the mean signal powers at each sensor, respectively, whereas the covariance matrices $\Sigma_{\mathbf{w}} \triangleq \mathrm{E}\left[\left(\mathbf{x}(n)-\mu_{\mathbf{w}}\right)(\mathbf{x}(n)-\right.$ $\left.\left.\mu_{\mathbf{w}}\right)^{T}\right]$ and $\Sigma_{\mathbf{s}} \triangleq \mathrm{E}\left[\left(\mathbf{x}(n)-\mu_{\mathbf{s}}\right)\left(\mathbf{x}(n)-\mu_{\mathbf{s}}\right)^{T}\right]$ represent the variability of the corresponding power estimates being reported by the sensors. At the BS, and because of the disturbances that may appear due to propagation through the reporting channel, we will assume that power measurements under hypothesis $\mathcal{H}_{0}$ are i.i.d. with some common variability $\sigma_{w}^{2}$ in such a way that $\Sigma_{\mathbf{w}}=\sigma_{w}^{2} \mathbf{I}_{K}$ for some unknown $\sigma_{w}^{2}$, and $\mathbf{I}_{K}$ the $(K \times K)$ identity matrix. Note also that both $\mu_{\mathbf{s}}$ and $\Sigma_{\mathbf{s}}$ depend on the characteristics of the random waveform being emitted by the PU and its position with respect to the $K$ sensors. Finally, and for the sake of clarity, we can express the signal model at the BS to be Gaussian distributed as follows:

$$
\begin{aligned}
& \mathcal{H}_{0}: \mathbf{x}(n) \sim \mathcal{N}\left(\mu_{0}, \Sigma_{0}\right) \\
& \mathcal{H}_{1}: \mathbf{x}(n) \sim \mathcal{N}\left(\mu_{1}, \Sigma_{1}\right)
\end{aligned}
$$

where $\mu_{0} \triangleq \mu_{\mathbf{w}}$ and $\Sigma_{0} \triangleq \Sigma_{\mathbf{w}}$ under the $\mathcal{H}_{0}$ hypothesis, whereas $\mu_{1} \triangleq \mu_{\mathbf{s}}+\mu_{\mathbf{w}}$ and $\Sigma_{1} \triangleq \Sigma_{\mathbf{s}}+\Sigma_{\mathbf{w}}$ under the $\mathcal{H}_{1}$ hypothesis. In practice, $\Sigma_{\mathbf{s}}$ will depart from a diagonal matrix, and the correlation represented by non-diagonal elements in $\Sigma_{s}$ will typically indicate the presence of correlated shadowing effects in the received PU signal strengths [10].

\section{Signature-based problem formulation}

\subsection{Preliminaries}

For an improved sensing performance, intuition suggests that the detection rule should rely on the observations of active sensors, thus discarding observations from the rest of inactive sensors. This approach can be understood as a kind of rank reduction method, whereby removing the most noisy dimensions of the received signal subspace leads to a significant improvement of the overall signalto-noise ratio. Moreover, since active sensors are typically located close to the PU, and also close to each other forming a spatial cluster, this side information should also be considered in the design of the detector. It is for this reason that one of the key points of this paper is the identification of the set of active sensors, a purpose that will be achieved through the help of model order selection techniques and the spatial structure of the neighboring sensors. To this end, we propose a structured signal model based on the concept of spatial signatures. For the case of the $i$ th sensor, its signature is a vector that contains the attenuation terms to all the $K$ sensors of the network, in such a way as if a signal source was located at the $i$ th sensor position. Thus, the $i$ th signature is a $(K \times 1)$ vector $\mathbf{h}_{i}$ as follows:

$$
\mathbf{h}_{i} \triangleq\left[h\left(d_{1, i}\right), \ldots, h\left(d_{i-1, i}\right), 1, h\left(d_{i+1, i}\right), \ldots, h\left(d_{K, i}\right)\right]^{T} .
$$

where $h\left(d_{i, j}\right)=d_{i, j}^{-\beta}$ takes into account the deterministic attenuation loss due to the distance between $i$ th and the $j$ th sensor locations, with $\beta$ as the known path loss exponent. We assume herein, similarly to [25], that the BS has complete knowledge of the sensors' positions in the network.

\subsection{Full-structured signal model}

The signatures of all $K$ sensors can be stacked into the so-called signatures matrix $\mathbf{H} \triangleq\left[\mathbf{h}_{1}, \mathbf{h}_{2} \ldots, \mathbf{h}_{K}\right]$, which is typically a full-rank $(K \times K)$ matrix having $K$ signatures as columns. Based on this formulation, the PU power levels received at any of the sensors can be expressed as a linear combination of the network signatures. That is to say, the values of $\mathbf{s}(n)$ in (4) can be expressed as $\mathbf{s}(n)=\mathbf{H a}(n)$, thus making explicit the role played by the spatial structure of the network, through the dependence on $\mathbf{H}$. Then, the signal model in (4) can be rewritten as:

$$
\begin{aligned}
& \mathcal{H}_{0}: \mathbf{x}(n)=\mathbf{w}(n) \\
& \mathcal{H}_{1}: \mathbf{x}(n)=\mathbf{H a}(n)+\mathbf{w}(n)
\end{aligned}
$$

where $\mathbf{a}(n) \sim \mathcal{N}\left(\mu_{\mathbf{a}}, \Sigma_{\mathbf{a}}\right)$ is a $(K \times 1)$ vector containing the random weights of each signature onto the received signal. That is to say, the elements within $\mathbf{a}(n)$ quantify the importance of each of the sensor signatures in the reconstruction of the signal field emitted by the PU. Therefore, by selecting the largest weights, we are actually choosing the most relevant sensors on the basis of their physical proximity to the PU. The closer the sensor is located to the $\mathrm{PU}$, the more aligned will be its signature vector to $\mathbf{s}(n)$, and thus, the larger the weight assigned to this signature. Using this linear combination of signatures in (7), we are 
taking into account both the distances between neighboring sensors and the location of the sensors with respect to the PU, thus fully exploiting the spatial information contained within the received signals. From a statistical point of view, the only difference with respect to the conventional unstructured signal model in (4) to (5) is that now, a specific structure is imposed onto both $\mu_{1}$ and $\Sigma_{1}$, with $\mu_{1}=\mathbf{H} \mu_{\mathbf{a}}+\mu_{\mathbf{w}}$ and $\Sigma_{1}=\mathbf{H} \Sigma_{\mathbf{a}} \mathbf{H}^{T}+\sigma_{w}^{2} \mathbf{I}_{K}$. Finally, once we have the signal model with the embedded spatial structure, the next step will be to select the relevant signatures contributing to the received signal, a topic that is discussed in Section 3.3.

\subsection{Rank-reduced structured signal model}

The PU will typically appear at an unknown and random position, and it will be surrounded by a given number of $L \leq K$ active sensors. In these circumstances, and in order to improve the spectrum sensing detection performance, we need to select the relevant signatures of active sensors so that the rest of $K-L$ signatures can reasonably be ignored. In some sense, we are in front of a detection problem where it is convenient to use a rank-reduced version of the signal model in (7). To do so, we will select the $L$ most relevant signatures using model order selection techniques [30]. Once we select the set of $L$ active sensors, their signatures will be stacked into a truncated $(K \times L)$ matrix $\mathbf{H}_{L}$. Similarly, the selected weights will be stacked into a $(L \times 1)$ vector $\mathbf{a}_{L}(n)$, which is the reduced version of vector $\mathbf{a}(n)$ in (7). The resulting rank-reduced signal model can be written as:

$$
\begin{aligned}
& \mathcal{H}_{0}: \mathbf{x}(n)=\mathbf{w}(n) \\
& \mathcal{H}_{1}: \mathbf{x}(n)=\mathbf{H}_{L} \mathbf{a}_{L}(n)+\mathbf{w}(n)
\end{aligned}
$$

where the random weights $\mathbf{a}_{L}(n)$ continue to be Gaussian distributed with $\mathbf{a}_{L}(n) \sim \mathcal{N}\left(\mu_{\mathbf{a}_{L}}, \Sigma_{\mathbf{a}_{L}}\right)$. Therefore, the difference with respect to the full structured model in (7) is that now, we have $\mu_{1}=\mathbf{H}_{L} \mu_{\mathbf{a}_{L}}+\mu_{\mathbf{w}}$ and $\Sigma_{1}=\mathbf{H}_{L} \Sigma_{\mathbf{a}_{L}} \mathbf{H}_{L}^{T}+\sigma_{w}^{2} \mathbf{I}_{K}$, both depending on the unknown model order $L$. It is important to remark that in addition to the spatial information provided by the use of spatial signatures, the rank-reduced version of matrix $\mathbf{H}$ will indeed allow us to benefit from an equivalent SNR gain by removing those subspace dimensions where the noise contribution is the dominant effect [31].

\section{Detection algorithms}

In our spectrum sensing detection problem, there are unknown parameters under both hypotheses that prevent us from adopting the well-known Neyman-Pearson detector. This obstacle is typically circumvented by adopting the GLRT approach, whereby the unknown parameters are substituted by their maximum likelihood estimates resulting in a simple and asymptotically optimal detector [32]. The main drawback, however, occurs when the dimension of the unknown signal vector (i.e., the model order) is unknown [33]. This situation occurs in the signal model (8), where the model order (i.e., the number of $L$ active sensors) is actually unknown, and thus, we cannot use the GLRT in a straightforward manner. Instead, we need to modify the GLRT in order to determine the appropriate value for $L$ to be used, a task that can be done using model order selection techniques [30]. In Section 4.1, we will derive the traditional detector based on the conventional GLRT, which will be used as a benchmark. Since no spatial information is considered in this detector, it will be referred to as the unstructured GLRT. Later on, in Section 4.2, we will derive an improved detector that incorporates both spatial information and the minimum description length (MDL) criterion for carrying out the model order selection. For the sake of clarity, this latter detector will be referred to as the structured GLRT.

\subsection{Unstructured GLRT}

In the original detection problem to be solved in (5), we need to estimate the unknowns $\left\{\mu_{0}, \Sigma_{0}\right\}$ under hypothesis $\mathcal{H}_{0}$, as well as the unknowns $\left\{\mu_{1}, \Sigma_{\mathbf{1}}\right\}$ under hypothesis $\mathcal{H}_{1}$. To do so, we will assume that the BS has available the measurements of $K$ sensors for $N$ consecutive sensing periods which are stacked into the $(K \times N)$ matrix $\mathbf{X} \triangleq[\mathbf{x}(1), \ldots, \mathbf{x}(n)]$. In these circumstances, the expression for the traditional or unstructured GLRT (UG) can be written as:

$$
\Lambda_{\mathrm{UG}}(\mathbf{X})=\frac{\max _{\mu_{0}, \Sigma_{0}} f\left(\mathbf{X} ; \mu_{0}, \Sigma_{0}\right)}{\max _{\mu_{1}, \Sigma_{1}} f\left(\mathbf{X} ; \mu_{1}, \Sigma_{1}\right)} \gtrless_{\mathcal{H}_{1}}^{\mathcal{H}_{0}} \gamma
$$

where $\gamma$ is a threshold that determines a given probability of false alarm. Following the GLRT approach, the values of the unknown parameters required in (9) are substituted by their maximum likelihood estimates (MLE). For the unknown mean vector $\mu_{1}$, its MLE can easily be found as:

$$
\hat{\mu}_{1}=\overline{\mathbf{x}} \triangleq \frac{1}{N} \sum_{n=1}^{N} \mathbf{x}(n) .
$$

Regarding the unknown covariance matrix $\Sigma_{1}$, its MLE can be written as [12, Lemma 3.2.1]:

$$
\hat{\Sigma}_{1}=\hat{\Sigma}_{\mathbf{x}}=\hat{\mathbf{R}}_{\mathbf{x}}-\overline{\mathbf{x}} \overline{\mathbf{x}}^{T}
$$

where $\hat{\mathbf{R}}_{\mathbf{x}} \triangleq \frac{1}{N} \sum_{n=1}^{N} \mathbf{x}(n) \mathbf{x}^{T}(n)$. It is interesting to note that the correlation matrix $\hat{\mathbf{R}}_{\mathbf{x}}$ and the mean vector $\overline{\mathbf{x}}$ are the sufficient statistics under hypothesis $\mathcal{H}_{1}$. Similarly under hypothesis $\mathcal{H}_{0}$, the MLE of $\mu_{0}$ can be obtained as $\hat{\mu}_{0}=\overline{\mathbf{x}}$, and the ML estimate of $\sigma_{w}^{2}$ can be found as:

$$
\hat{\sigma}_{w}^{2}=\frac{1}{K} \operatorname{Tr}\left(\hat{\Sigma}_{\mathbf{x}}\right)
$$


where $\operatorname{Tr}(\cdot)$ is the trace operator. Replacing all the unknowns with their estimates, and after some mathematical manipulations, the final expression for the unstructured GLRT in (9) turns out to be given by:

$$
\Lambda_{\mathrm{UG}}(\mathbf{X})=\frac{\left|\hat{\Sigma}_{\mathbf{x}}\right|}{\left[\frac{1}{K} \operatorname{Tr}\left(\hat{\Sigma}_{\mathbf{x}}\right)\right]^{K}} \gtrless_{\mathcal{H}_{1}}^{\mathcal{H}_{0}} \gamma .
$$

The test statistic in (13), which does not take into account any spatial information, is nothing but the traditional Mauchly's sphericity test [12, Chap 10]. The detector operates with the full sample covariance matrix $\hat{\Sigma}_{\mathbf{x}}$, and it neither considers the relevance of active sensors nor the spatial structure as side information. A modified sphericity test has been proposed in $[34,35]$ by exploiting the fact that the signal covariance matrix may be of lowrank dimensionality. However, the resulting detector does not really exploit the spatial structure of neighboring sensors. In that sense, the performance of this unstructured sphericity test-based detector can be further improved by incorporating the proposed concept of spatial signatures, which acts as an additional side information and allows us to select only those particular observations reported by active sensors. This novel feature will be introduced next in Section 4.2.

\subsection{Structured GLRT with spatial information}

As we have already mentioned in Section 3.3, the key point in the proposed rank-reduced signal model in (8) is the determination of the spatial model order $L$. Since $L \leq K$, the detector with spatial information can operate with a reduced signal subspace by rejecting those dimensions (i.e., those spatial signatures) where the PU signal contribution is almost negligible. Therefore, some performance gain is expected compared to traditional unstructured signal detectors. The process of determining the optimal $L$ is coupled with the one of signal detection, and this leads to the following structured GLRT (SG) detector:

$$
\begin{aligned}
\Lambda_{\mathrm{SG}}^{\prime}(\mathbf{X}) & =\max _{1 \leq l \leq K} \Lambda_{\mathrm{SG}, l}(\mathbf{X}) \\
& =\max _{1 \leq l \leq K}\left\{\frac{\max _{\mu_{0}, \Sigma_{0}} f\left(\mathbf{X} ; \mu_{0}, \Sigma_{0}\right)}{\max _{\mu_{1, l}, \Sigma_{1, l}} f\left(\mathbf{X} ; \mu_{1, l}, \Sigma_{1, l}\right)}\right\} \gtrless_{\mathcal{H}_{1}}^{\mathcal{H}_{0}} \gamma .
\end{aligned}
$$

The denominator in (14) is the likelihood function of the observation under hypothesis $\mathcal{H}_{1}$, which includes the unknown model order $L$ as an additional parameter to be determined by searching the maximum of the GLRT from $l=1, \ldots, K$. As a result, the mean vector and the covariance matrix under hypothesis $\mathcal{H}_{1}$ now depend on the tentative model order $l$, according to the rank-reduced spatial structure considered in (8). That is, for the mean vector, we have $\mu_{1, l} \triangleq \mathbf{H}_{l} \mu_{\mathbf{a}_{l}}+\mu_{\mathbf{w}}$ and for the covariance matrix $\Sigma_{1, l} \triangleq \mathbf{H}_{l} \Sigma_{\mathbf{a}_{l}} \mathbf{H}_{l}^{T}+\sigma_{w}^{2} \mathbf{I}_{K}$.

The problem with (14) is that the inner GLRT $\Lambda_{\mathrm{SG}, l}(\mathbf{X})$ monotonically increases as a function of $l$, and thus, the result to the overall search process is always given by the test statistic having the maximum model order $l=$ $K$. This occurs because the tentative probability density functions $f\left(\mathbf{X} ; \mu_{1, l}, \Sigma_{\mathbf{1}, \mathbf{l}}\right)$ are a set of nested families. The net effect is that the test statistic will always overestimate the actual model order, thus including dimensions of the signal subspace where there is almost no signal, but only noise is present. This results in a reduction of the power of the detector to produce a desired result [33]. This problem will occur whenever the number of signal components is unknown, and that is why the conventional GLRT poses some limitations in this type of nested or model order-based detection problems. To cope with this problem, several model order selection criteria have been proposed in the literature. They are based on the incorporation of an additional penalty function, which prevents the likelihood function to increase without bound when increasing the model order [30]. Herein, we will consider the well-known MDL criterion. With the help of this selection technique, both the estimation of the true model order $L$ and the evaluation of the GLRT can be done jointly. The detector combining the structured GLRT and the MDL can be expressed as follows:

$$
\Lambda_{\mathrm{SG}}(\mathbf{X})=\min _{1 \leq l \leq K}\left\{l \log K-2 \log \Lambda_{\mathrm{SG}, l}(\mathbf{X})\right\} \gtrless_{\mathcal{H}_{1}}^{\mathcal{H}_{0}} \gamma
$$

where $l \log K$ is a penalty function that prevents the GLRT statistic to monotically increase with increasing model orders. In (15), $\Lambda_{\mathrm{SG}, l}(\mathbf{X})$ stands for the structured GLRT statistic while considering $\hat{L}=l$ as the tentative model order, whose likelihood functions under both $\mathcal{H}_{1}$ and $\mathcal{H}_{0}$ hypotheses will be derived in Section 4.2.1. Later on, in Section 4.2.2, we will propose an algorithm to evaluate the structured GLRT in (15) and take advantage of the available spatial information embedded onto the signatures matrix.

\subsubsection{Derivation of the structured GLRT for the tentative model order $\hat{L}=I$}

In this section, we will derive the expression for the structured GLRT $\Lambda_{\mathrm{SG}, l}(\mathbf{X})$ required in (15), which assumes a tentative model order $\hat{L}=l$ for the parameters $\left\{\mu_{1, l}, \Sigma_{1, l}\right\}$ in the likelihood function under hypothesis $\mathcal{H}_{1}$. Bearing in mind the Gaussian nature of the received measurements 
at the BS, as already introduced in Section 3.3, the likelihood function in the denominator of $\Lambda_{\mathrm{SG}, l}(\mathbf{X})$ in (14) is given by:

$$
f\left(\mathbf{X} ; \boldsymbol{\mu}_{1, l}, \Sigma_{1, l}\right)=\left|\Sigma_{1, l}\right|^{-\frac{N}{2}} \exp \left[-\frac{N}{2} \operatorname{tr}\left(\Sigma_{1, l}^{-1} \hat{\Sigma}_{\mathbf{x}, l}\right)\right]
$$

where the reduced rank spatial covariance matrix $\Sigma_{\mathbf{a}_{l}}$ is embedded into $\Sigma_{1, l}$, since $\Sigma_{1, l}=\mathbf{H}_{l} \Sigma_{\mathbf{a}_{l}} \mathbf{H}_{l}^{T}+\sigma_{w_{1}}^{2} \mathbf{I}_{K}$. Regarding the sample covariance matrix $\hat{\Sigma}_{\mathbf{x}, l}$ in (16), it has the following expression:

$$
\hat{\Sigma}_{\mathbf{x}, l}=\frac{1}{N} \sum_{n=1}^{N}\left(\mathbf{x}(n)-\mathbf{H}_{l} \boldsymbol{\mu}_{\mathbf{a}_{l}}\right)\left(\mathbf{x}(n)-\mathbf{H}_{l} \boldsymbol{\mu}_{\mathbf{a}_{l}}\right)^{T}
$$

where the MLE of the unknown mean vector $\mu_{\mathbf{a}_{l}}$ is found as $\hat{\boldsymbol{\mu}}_{\mathbf{a}_{l}}=\left(\mathbf{H}_{l}^{T} \mathbf{H}_{l}\right)^{-1} \mathbf{H}_{l}^{T} \overline{\mathbf{x}}$, where $\overline{\mathbf{x}} \triangleq \frac{1}{N} \sum_{n=1}^{N} \mathbf{x}(n)$. Substituting $\hat{\mu}_{\mathbf{a}_{l}}$ into (17) and using [12, Lemma 3.2.1], we can write (17) as:

$$
\hat{\Sigma}_{\mathbf{x}, l}=\hat{\mathbf{R}}_{x}-\overline{\mathbf{x}} \overline{\mathbf{x}}^{T}+\left(\overline{\mathbf{x}}-\overline{\mathbf{x}}_{p, l}\right)\left(\overline{\mathbf{x}}-\overline{\mathbf{x}}_{p, l}\right)^{T}
$$

where $\overline{\mathbf{x}}_{p, l} \triangleq \mathbf{P}_{\mathbf{H}_{l}} \overline{\mathbf{x}}$, and $\mathbf{P}_{\mathbf{H}_{1}} \triangleq \mathbf{H}_{l}\left(\mathbf{H}_{l}^{T} \mathbf{H}_{l}\right)^{-1} \mathbf{H}_{l}^{T}$ is the projection matrix onto the $l$-dimensional subspace spanned by rank-reduced spatial signatures matrix $\mathbf{H}_{l}$. The vector $\overline{\mathbf{x}}_{p, l}$ is therefore the projected version of the mean vector $\overline{\mathbf{x}}$ onto the subspace spanned by the columns (i.e., signatures) of $\mathbf{H}_{l}$. Next, in order to find the MLE of $\Sigma_{\mathbf{a} l}$, we can apply the logarithm on both sides of (16), take the derivative w.r.t. $\Sigma_{\mathbf{a}_{l}}$, and equate to zero. By doing so, we get [36, Sec. 8.5 ],

$$
\hat{\Sigma}_{\mathbf{a}_{l}}=\mathbf{H}_{l}^{\dagger}\left[\hat{\Sigma}_{\mathbf{x}, l}-\hat{\sigma}_{w_{1}}^{2} \mathbf{I}_{K}\right] \mathbf{H}_{l}^{\dagger}
$$

where $\mathbf{H}_{l}^{\dagger} \triangleq\left(\mathbf{H}_{l}^{T} \mathbf{H}_{l}\right)^{-1} \mathbf{H}_{l}^{T}$ is the Moore-Penrose pseudoinverse, and $\hat{\sigma}_{w_{1}}^{2}$ is given by:

$$
\hat{\sigma}_{w_{1}}^{2}=\frac{\operatorname{Tr}\left[\mathbf{P}_{H_{l}}^{\perp} \hat{\Sigma}_{\mathbf{x}, l}\right]}{K-l+1}
$$

with $\mathbf{P}_{\mathbf{H}_{l}}^{\perp} \triangleq \mathbf{I}_{K}-\mathbf{P}_{\mathbf{H}_{l}}$, the orthogonal projection matrix of $\mathbf{P}_{\mathbf{H}_{l}}$. In (20), the variance $\sigma_{w_{1}}^{2}$ of power estimates at the $\mathrm{BS}$ is estimated using the projected version of the observation vector $\mathbf{x}(n)$ onto the noise subspace, since $\mathbf{P}_{H_{l}}^{\perp} \hat{\Sigma}_{x}=$ $\mathbf{P}_{H_{l}}^{\perp}\left(\hat{\mathbf{R}}_{x}-\overline{\mathbf{x}} \overline{\mathbf{x}}^{T}\right)=\frac{1}{N} \sum_{n=1}^{N}\left[\mathbf{P}_{H_{l}}^{\perp} \mathbf{x}(n)\right]\left[\mathbf{P}_{H_{l}}^{\perp} \mathbf{x}(n)\right]^{T}-$ $\mathbf{P}_{H_{l}}^{\perp} \overline{\mathbf{x}}\left(\mathbf{P}_{H_{l}}^{\perp} \overline{\mathbf{x}}\right)^{T}$. Consequently, for the overall covariance matrix $\Sigma_{1, l}$ in (16) we have,

$$
\hat{\Sigma}_{1, l}=\mathbf{P}_{\mathbf{H}_{l}}\left[\hat{\Sigma}_{\mathbf{x}, l}-\hat{\sigma}_{w_{1}}^{2} \mathbf{I}_{K}\right] \mathbf{P}_{\mathbf{H}_{l}}+\hat{\sigma}_{w_{1}}^{2} \mathbf{I}_{K}
$$

which can also be written as,

$$
\hat{\Sigma}_{1, l}=\mathbf{P}_{\mathbf{H}_{l}} \hat{\Sigma}_{\mathbf{x}, l} \mathbf{P}_{\mathbf{H}_{l}}+\frac{1}{K-l+1} \operatorname{Tr}\left[\mathbf{P}_{\mathbf{H}_{l}}^{\perp} \hat{\Sigma}_{\mathbf{x}, l}\right] \mathbf{P}_{\mathbf{H}_{l}}^{\perp} .
$$

On the other hand, under hypothesis $\mathcal{H}_{0}$, we need to determine the unknown parameters $\left\{\mu_{0}, \Sigma_{0}\right\}$ required by likelihood function in the numerator of $\Lambda_{\mathrm{SG}, l}(\mathbf{X})$ in (14). Regarding the MLE of $\Sigma_{0}=\sigma_{w_{0}}^{2} \mathbf{I}_{K}$, it can be obtained from $\hat{\sigma}_{w_{0}}^{2}=\frac{1}{K} \operatorname{Tr}\left(\hat{\mathbf{R}}_{\mathbf{x}}-\overline{\mathbf{x}} \overline{\mathbf{x}}^{T}\right) \simeq \frac{1}{K} \operatorname{Tr}\left(\hat{\Sigma}_{\mathbf{x}, l}\right)$, where we already used the fact that $\hat{\mu}_{0}=\overline{\mathbf{x}}$. With these results in mind, we can obtain the expression for the structured GLRT with tentative model order $\hat{L}=l$ as:

$$
\Lambda_{\mathrm{SG}, l}(\mathbf{X}) \simeq \frac{\left|\hat{\sigma}_{w_{0}}^{2} \mathbf{I}\right|^{-\frac{N}{2}} \exp \left[-\frac{N}{2}\right]}{\left|\hat{\Sigma}_{l}\right|^{-\frac{N}{2}} \exp \left[-\frac{N}{2}\right]}=\frac{\left|\hat{\Sigma}_{1, l}\right|^{\frac{N}{2}}}{\hat{\sigma}_{w_{0}}^{K N}} .
$$

Substituting $\hat{\Sigma}_{1, l}=\mathbf{P}_{\mathbf{H}_{l}} \hat{\Sigma}_{\mathbf{x}, l} \mathbf{P}_{\mathbf{H}_{l}}+\hat{\sigma}_{w_{1}}^{2} \mathbf{P}_{\mathbf{H}_{l}}^{\perp}$ and $\hat{\sigma}_{w_{0}}^{2}=$ $\frac{1}{K} \operatorname{Tr}\left(\hat{\Sigma}_{\mathbf{x}, l}\right)$, and after some mathematical manipulations, (23) can equivalently be expressed as:

$$
\Lambda_{\mathrm{SG}, l}(\mathbf{X}) \simeq \frac{\left|\mathbf{P}_{\mathbf{H}_{l}} \hat{\Sigma}_{\mathbf{x}, l} \mathbf{P}_{\mathbf{H}_{l}}+\frac{1}{K-l+1} \operatorname{Tr}\left(\mathbf{P}_{\mathbf{H}_{l}}^{\perp} \hat{\Sigma}_{\mathbf{x}, l}\right) \mathbf{P}_{\mathbf{H}_{l}}^{\perp}\right|^{\frac{N}{2}}}{\left[\frac{1}{K} \operatorname{Tr}\left(\hat{\Sigma}_{\mathbf{x}, l}\right)\right]^{\frac{K N}{2}}} .
$$

The expression in (24) provides a closed-form expression for the structured GLRT with tentative model order $\hat{L}=l$. The main feature of this expression is that it selects the most relevant spatial signatures, and then on the basis of these signatures, it reduces the rank of the measurements covariance matrix, $\Sigma_{\mathbf{x}}$. This statement can be explained by defining $\Psi_{l} \triangleq \mathbf{P}_{\mathbf{H}_{l}} \hat{\Sigma}_{\mathbf{x}, l} \mathbf{P}_{\mathbf{H}_{l}}$ and noticing that $\Psi_{l}=\mathbf{P}_{\mathbf{H}_{l}} \hat{\mathbf{R}}_{\mathbf{x}} \mathbf{P}_{\mathbf{H}_{l}}-\mathbf{P}_{\mathbf{H}_{l}} \overline{\mathbf{x}} \overline{\mathbf{x}}^{T} \mathbf{P}_{\mathbf{H}_{l}}$, which can be equivalently expressed using the properties of projection matrices as $\Psi_{l} \triangleq \frac{1}{N} \sum_{n=0}^{N}\left[\mathbf{P}_{\mathbf{H}_{l}} \mathbf{x}(n)\right]\left[\mathbf{P}_{\mathbf{H}_{l}} \mathbf{x}(n)\right]^{T}-\left[\mathbf{P}_{\mathbf{H}_{l}} \overline{\mathbf{x}}\right]\left[\mathbf{P}_{\mathbf{H}_{l}} \overline{\mathbf{x}}\right]^{T}$. The expression of $\Psi_{l}$ clearly shows that it is indeed the sample covariance matrix of a vector achieved by projecting the received observations $\mathbf{x}(n)$ onto the specific subspace being spanned by the signatures of active sensors. This will indeed result in an SNR gain due to the projection of the observation vector onto a reduced dimensionality subspace.

\subsubsection{Implementation of the structured GLRT with MDL model order selection}

The next step is to substitute the expression in (24) into (15) and perform the joint PU signal detection and model 
Algorithm 1 MDL-based structured GLRT.

1. With the received observation vectors $\mathbf{x}(n), n=1,2, \cdots, N$, we calculate:

$$
\hat{\mathbf{R}}_{\mathbf{x}}=\frac{1}{N} \sum_{n=1}^{N} \mathbf{x}(n) \mathbf{x}^{T}(n), \overline{\mathbf{x}}=\frac{1}{N} \sum_{n=1}^{N} \mathbf{x}(n) \text { and } \hat{\Sigma}_{\mathbf{x}, 0}=\hat{\mathbf{R}}_{\mathbf{x}}-\overline{\mathbf{x}} \overline{\mathbf{x}}^{T} .
$$

2. Find $\hat{\boldsymbol{\mu}}_{\mathbf{a}}=\mathbf{H}^{-1} \overline{\mathbf{x}}$.

3. Take the absolute values $\left|\hat{\boldsymbol{\mu}}_{\mathbf{a}}\right|$ and sort $\left|\hat{\boldsymbol{\mu}}_{\mathbf{a}}\right|$ in descending order, which results in $\tilde{\boldsymbol{\mu}}_{\mathbf{a}}=\operatorname{sort}\left(\left|\hat{\boldsymbol{\mu}}_{\mathbf{a}}\right|\right)$.

4. Reorder the signature vectors in $\mathbf{H}$ according to the sorted $\tilde{\boldsymbol{\mu}}_{\mathbf{a}}$, to get $\tilde{\mathbf{H}}$.

5. Implement the detector as:

- Initialize $\mathbf{t}=\langle\rangle$ and $l=1$.

- while $l \leq K$ do:

- Set $\mu_{\mathbf{a}_{l}}=\tilde{\mu}_{\mathbf{a}}(1: l)$.

- Set $\mathbf{H}_{l}=\widetilde{\mathbf{H}}(1: l)$.

- Calculate $\mathbf{P}_{\mathbf{H}_{l}}=\mathbf{H}_{l}\left(\mathbf{H}_{l}^{T} \mathbf{H}_{l}\right)^{-1} \mathbf{H}_{l}^{T}$.

- Calculate $\overline{\mathbf{x}}_{p, l}=\mathbf{P}_{\mathbf{H}_{l}} \overline{\mathbf{x}}$.

- Calculate $\hat{\Sigma}_{\mathbf{x}, l}$ in (18) as $\hat{\Sigma}_{\mathbf{x}, l}=\hat{\Sigma}_{\mathbf{x}, 0}+\left(\overline{\mathbf{x}}-\overline{\mathbf{x}}_{p, l}\right)\left(\overline{\mathbf{x}}-\overline{\mathbf{x}}_{p, l}\right)^{T}$.

- Calculate $\Lambda_{\mathrm{SG}, l}(\mathbf{X})$ in (24).

- Push the result of $l \log K-2 \log \Lambda_{\mathrm{SG}, l}(\mathbf{X})$ onto the vector $\mathbf{t}$.

$-l=l+1$.

- end while

- $\Lambda_{\mathrm{SG}}(\mathbf{X})=\min \{\mathbf{t}\} \gtrless_{\mathcal{H}_{1}}^{\mathcal{H}_{0}} \gamma$ as in (15).

order selection from $l=1, \ldots, K$. To do so, we summarize the implementation of the resulting detector in the pseudocode description indicated in Algorithm 1.

\section{Improved estimation of the covariance matrix}

Both the unstructured and the structured GLRT detectors presented in this paper are found to be based on the determinant of covariance matrices, which are typically estimated through the sample covariance, as in (11) and (18), respectively. Therefore, and although it is often taken for granted, a critical requirement for the GLRT detectors under study is that, the sample covariance matrices must be non-singular and positive definite. To this end, we have to make sure that the number of available observations at the $\mathrm{BS}$, given by $N$, is much larger than the number of sensors $K$ (i.e., $N \gg K$ ). However, in many sensor network deployments, we typically have a very large $K$, and thus, using a number of samples greater than $K$ is a requirement that is difficult to fulfill in practice. In these circumstances, it is therefore needed to estimate the covariance matrix with fewer samples while keeping a reasonable detection performance. Stein in [37], introduced the concept of shrinkage applied to high-dimensional estimators, and he derived the striking result that the performance of MLE can always be improved upon by shrinking with a given factor $\alpha$ (shrinkage intensity). This improved covariance estimator is well-conditioned and always positive definite, even for small sample sizes [21]. The basic principle of shrinking estimators is to shrink the variation of the eigenvalues in the sample covariance matrix, proceeding as follows:

$$
\breve{\Sigma}_{\mathbf{x}}=\alpha \mathbf{F}_{0}+(1-\alpha) \hat{\Sigma}_{\mathbf{x}}, 0 \leq \alpha \leq 1
$$

where $\mathbf{F}_{0}$ is the target matrix, which is chosen to be positive definite (and therefore nonsingular) and wellconditioned, and we assume it herein to be given by $\mathbf{F}_{0}=\frac{1}{K} \operatorname{Tr}\left(\hat{\Sigma}_{\mathbf{x}}\right) \mathbf{I}_{K}$. The interested reader can find further details about the target matrix in [21]. Now, for the expression in (25), we need to choose an appropriate $\alpha$, the shrinkage intensity parameter. In [38], the authors discuss shrinkage methods that calculate the intensity parameter on the basis of received observations. They present what they call an oracle approximating shrinkage (OAS) estimator, which is an iterative method presented in Algorithm 2. 


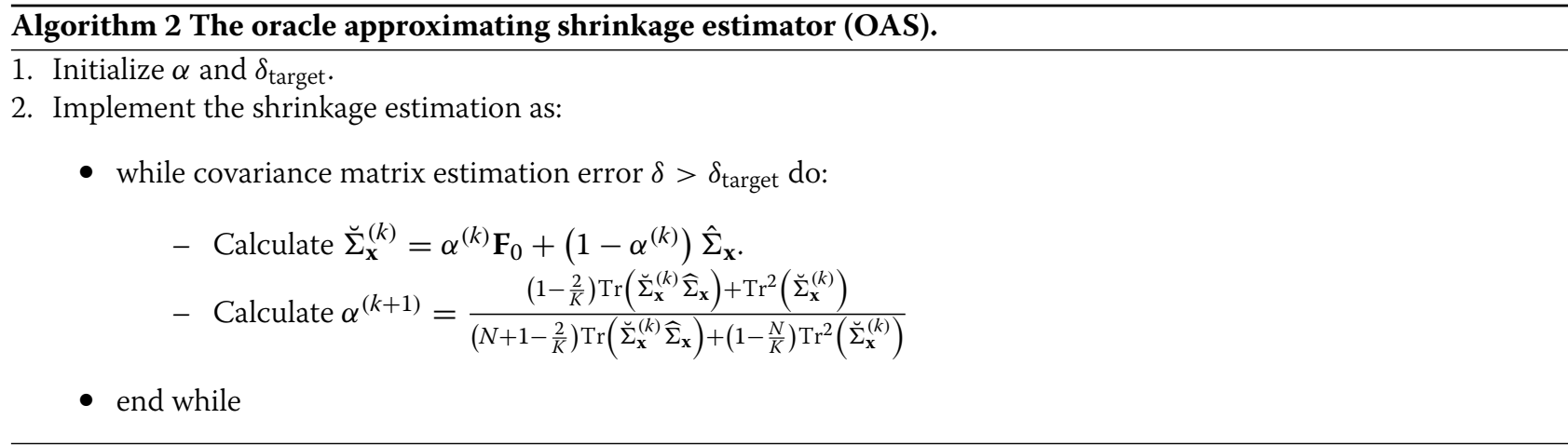

In Algorithm 2, $\delta_{\text {Target }}$ represents a specified threshold for the covariance matrix estimation error. The algorithm stops once the estimation error turns out to be less than this threshold. Once the algorithm has converged, it reaches the following stable value of the shrinkage parameter,

$$
\alpha_{\text {approx }} \approx \min \left\{\frac{\left(1-\frac{2}{K}\right) \operatorname{Tr}\left(\hat{\Sigma}_{\mathbf{x}}^{2}\right)+\operatorname{Tr}^{2}\left(\hat{\Sigma}_{\mathbf{x}}\right)}{\left(N+1-\frac{2}{K}\right)\left[\operatorname{Tr}\left(\hat{\Sigma}_{\mathbf{x}}^{2}\right)-\frac{1}{K} \operatorname{Tr}^{2}\left(\hat{\Sigma}_{\mathbf{x}}\right)\right]}, 1\right\}
$$

In our detection schemes, we will use $\alpha_{\text {approx }}$ in (26), the approximate value of the shrinkage parameter in the covariance matrix estimation process indicated in (25).

\section{Simulation results}

The motivation of this section is to assess the performance of the proposed structured GLRT detector in (15) and (24), which takes advantage of the novel concept of spatial signatures introduced in Section 3 and whose implementation is described in the pseudocode description of Algorithm 1. For the analysis to be conducted herein, we consider a wireless sensor network with a total of $K=30$ sensors deployed in a squared field. The sensors are randomly placed within the field following a uniform distribution, and we assume that the PU appears at an unknown position. We have tested the detectors considered in this paper for many different uniformly distributed topologies of $K$ sensors, and we have found that the results have similar characteristics for different random topologies.

For the signal generation, we are assuming a quasistatic block-fading channel in which both the PU received power and the noise power at each sensor do remain constant within the observation interval of $N$ measurements. For a given observation interval, the PU received power at sensor $i$ is given by $P_{\epsilon, i}=P_{0} d_{i}^{-\beta} 10^{X_{\sigma} / 10}$, where $P_{0}$ is the power at a reference distance from the $\mathrm{PU}, \beta$ is the signal decay exponent with typical values from 2 to $5, d_{i}$ is the
Euclidean distance between the PU and sensor $i$, and $X_{\sigma}$ is the value of the log-normal shadowing. From one observation interval to the following, we allow the shadowing to vary according to $X_{\sigma} \sim \mathcal{N}\left(0, \sigma_{X}^{2}\right)$, with $\sigma_{X}$ as the standard deviation [26]. Regarding the noise power at sensor $i$, we are assuming $\sigma_{\varepsilon}^{2}=10^{\Delta_{\sigma} / 10}$ for all sensors, with $\Delta_{\sigma}$ modeling the log-normal noise uncertainty as $\Delta_{\sigma} \sim \mathcal{N}\left(0, \sigma_{\Delta_{\sigma}}^{2}\right)$, from one observation interval to the following [39]. At the $\mathrm{BS}$, the variability of the received power measurements is given by $\sigma_{w}^{2}=\frac{2 \sigma_{\varepsilon}^{4}}{M}+\sigma_{f}^{2}$, where the first term represents the variability due to the sensors themselves, as indicated in (3), and the second term incorporates an additional disturbance $\sigma_{f}^{2}$ due to the noisy reporting links that connect sensors to the BS.

Regarding the assessment of the detectors being considered in this paper, we will analyze their performance with and without the shrinkage estimation through the use of receiver operating characteristic (ROC) curves. Although the ROC curves fully characterize the performance, it is also desirable to have a single and quantitative figure of merit in order to compare different detectors. This metric is typically the area under the ROC curve (AUC), which varies between 0.5 (poor performance) and 1 (good performance). The AUC is mathematically expressed as $\operatorname{AUC}(T)=\int_{0}^{1} P_{D}(T) d P_{F A}(T)$, where $T$ represents some specific detector, $P_{D}$ indicates the probability of detection, and $P_{F A}$ is the probability of false alarm. For the traditional unstructured GLRT in (9), the theoretical characterization of both $P_{D}$ and $P_{F A}$ (as well as the associated detection threshold $\gamma$ ) can be determined for an asymptotically large observation interval. In that asymptotic case, closed-form expressions can be found because the statistics of the GLRT can be well approximated by a chisquared $\left(\chi^{2}\right)$ distribution [32, Sec.6.5]. Unfortunately, this is not the case for the structured GLRT in (15), whose performance turns out to be coupled with that of the MDL model-order selection criterion, thus posing insurmountable obstacles to the derivation of a closed-form statistical characterization. In order to circumvent this limitation, we resort to the numerical evaluation of $P_{D}$ and $P_{F A}$ through the numerical computation of the ROC curve. 
To do so, we use the algorithm proposed in [40],which provides a computationally efficient method for determining the performance of a given detector in terms of $P_{D}$ as a function of $P_{F A}$. Once the ROC curve is available, we calculate the AUC curve on the basis of integrating the areas of small trapezoidal bins from the ROC curve. That is to say, $\operatorname{AUC}(T)=\sum_{\kappa} \bar{P}_{D}^{\kappa}(T) \Delta P_{F A}^{\kappa}(T)$, where $\Delta P_{F A}^{\kappa}(T)=P_{F A}^{\kappa+1}(T)-P_{F A}^{\kappa}(T)$ and $\bar{P}_{D}^{\kappa}(T)=\frac{P_{D}^{\kappa+1}(T)+P_{D}^{\kappa}(T)}{2}$.

\subsection{Experiment 1: ROC curves for the detection schemes} In Figure 1, we evaluate the ROC curves for the proposed detection schemes by setting the PU transmit power $P_{0}=-7 \mathrm{~dB}$ so that the mean received power in the sensor field turns out to be $-37 \mathrm{~dB}$. In Figure 1a, we present the results in the absence of noise power uncertainty (i.e., $\sigma_{\Delta_{\sigma}}=0 \mathrm{~dB}$ ), where two conclusions can be drawn. First, that the structured GLRT $\Lambda_{\mathrm{SG}}(\mathbf{X})$ clearly outperforms the unstructured GLRT $\Lambda_{\mathrm{UG}}(\mathbf{X})$ when no
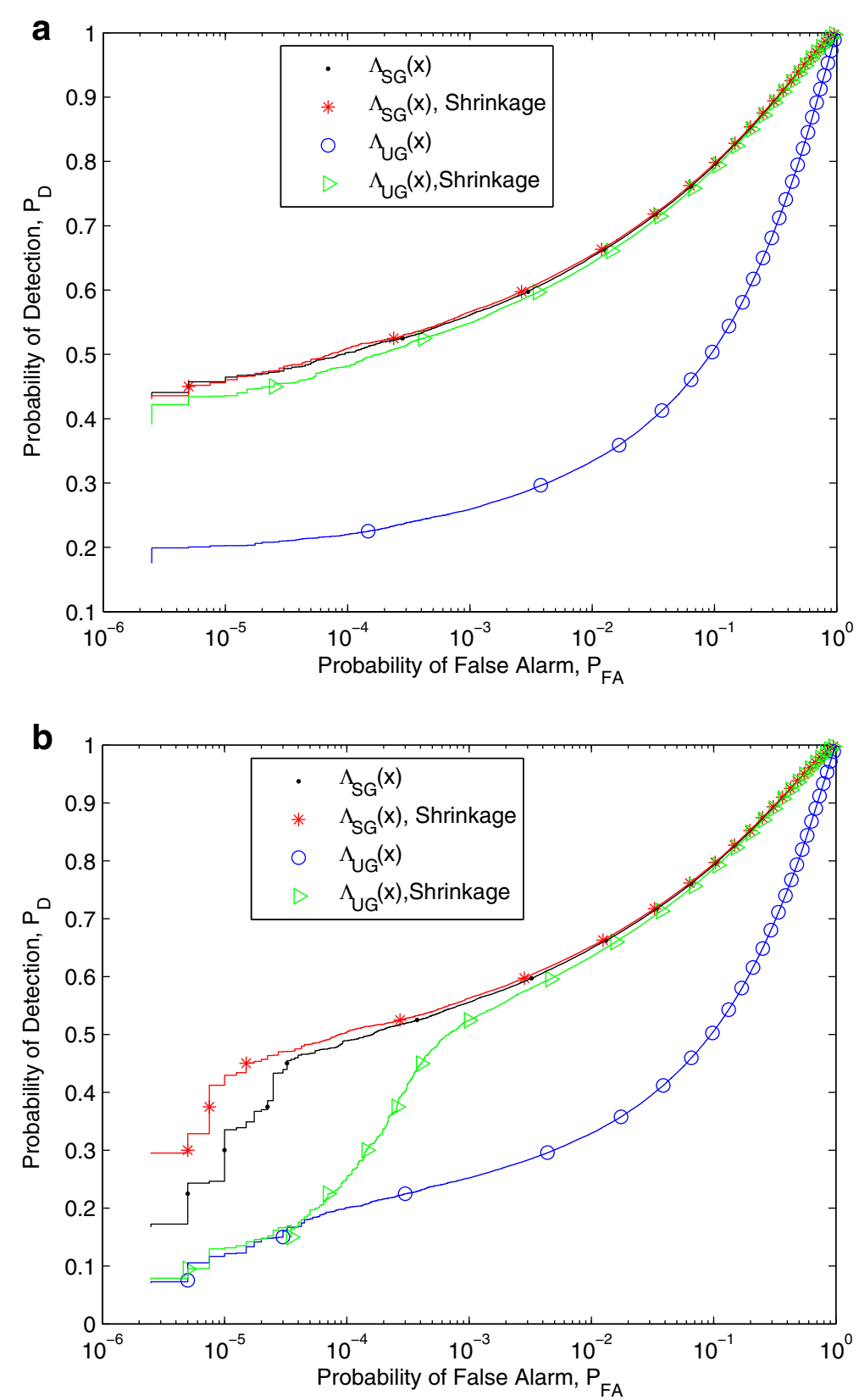

Figure 1 Receiver operating characteristic (ROC) curves: $\sigma_{X}=\mathbf{3} \mathrm{dB}, \boldsymbol{N}=\mathbf{1 0 0}, \boldsymbol{M}=\mathbf{2 5 0}, \boldsymbol{P}_{\mathbf{0}}=\mathbf{- 7} \mathrm{dB}$, and $\boldsymbol{\sigma}_{f}=\mathbf{0} \mathrm{dB}$. (a) ROC curves of $\sigma_{\Delta_{\sigma}}=0 \mathrm{~dB}$. (b) ROC curves of $\sigma_{\Delta_{\sigma}}=2 \mathrm{~dB}$. In this figure, we simulate the receiver operating characteristic (ROC) curves for the detectors discussed above. Details about this figure can be found in subsection with the heading Experiment 1 in Section 6. 
shrinkage is implemented. Second, that when shrinkage is implemented, the performance of the unstructured GLRT is boosted and becomes close to the one provided by the structured GLRT. This observation suggests that in the absence of noise power uncertainty, shrinkage has a similar effect to rank-reduction implemented by the use of spatial signatures. Interestingly, the situation changes when noise power uncertainty appears. This can be observed in Figure 1b, where we plot the ROC curves for the case of $\sigma_{\Delta_{\sigma}}=2 \mathrm{~dB}$. In that case, the unstructured GLRT $\Lambda_{\mathrm{UG}}(\mathbf{X})$ is severely degraded irrespective of whether shrinkage is implemented or not, whereas the proposed structured GLRT $\Lambda_{\mathrm{SG}}(\mathbf{X})$ is found to exhibit a more robust and superior performance, particularly for small $P_{F A}$.

\subsection{Experiment 2: Sensitivity to noise power uncertainty}

In Figure 2, we compare the AUC plots Xx of the detectors under study in order to further analyze the effects of noise power uncertainty preliminary highlighted in Experiment 1. To do so, and for the same parameters as in the previous experiment, we now let the noise power uncertainty range from $\sigma_{\Delta_{\sigma}}=0 \mathrm{~dB}$ to $\sigma_{\Delta_{\sigma}}=12 \mathrm{~dB}$. The AUC plots clearly show that the unstructured GLRT $\Lambda_{\mathrm{UG}}(\mathbf{X})$ with shrinkage estimation starts to degrade for noise power uncertainties greater than $\sigma_{\Delta_{\sigma}}=3 \mathrm{~dB}$. In contrast, the structured GLRT $\Lambda_{\mathrm{SG}}(\mathbf{X})$ (both with and without shrinkage) is able to cope with higher noise power uncertainties and provide on the order of a $15 \%$ to $20 \%$ improvement in terms of AUC in the range $\sigma_{\Delta_{\sigma}} \in[5.5,8.0] \mathrm{dB}$. Indeed, the impact of noise power uncertainty is even more severe in the case of low SNR, as suggested in [39], and $\Lambda_{\mathrm{SG}}(\mathbf{X})$ is able to counteract this situation by increasing the system's SNR by selecting the relevant samples of active sensors.
It is also found that the performance of different detection schemes improves by shrinkage estimation, though the improvement is very small in the case of the structured GLRT $\Lambda_{\mathrm{SG}}(\mathbf{X})$, for which the use of signatures already provides the required robustness to cope with harsh working conditions. In this experiment, we have also analyzed the impact of noise power uncertainty on a energy detector at a single node. We remark here that we selected a sensor that is located close to the PU, and it receives signal with high SNR. In spite of that, we can see that the performance of the energy detector at a single node is severely affected by noise uncertainty, thus confirming the advantages of the proposed approach of collaborating sensing with spatial information.

\subsection{Experiment 3: Sensitivity to shadowing in the channel between the PU and SUs}

In this experiment, we analyze the sensitivity to the shadowing present in the channel between the PU and SUs, quantified by the parameter $\sigma_{X}$, and for two different working conditions (i.e., low and high SNR). We first start with the low SNR scenario in Figure 3, where two different cases of noise power uncertainties are analyzed as a function of shadowing. These two cases correspond to $\sigma_{\Delta_{\sigma}}=0 \mathrm{~dB}$ in Figure 3a and $\sigma_{\Delta_{\sigma}}=5 \mathrm{~dB}$ in Figure 3b. As already highlighted in previous experiments, the results in the presence of shadowing also show a superior performance for the structured GLRT $\Lambda_{\mathrm{SG}}(\mathbf{X})$, particularly for high noise power uncertainties, i.e., as in Figure 3b. Interestingly, the detection schemes under analysis are found to perform better as the shadow fading becomes more variable (i.e., higher $\sigma_{X}$ ). This is because of the heavy-tailed distribution of the PU-received power in the presence of log-normally distributed shadow fading,

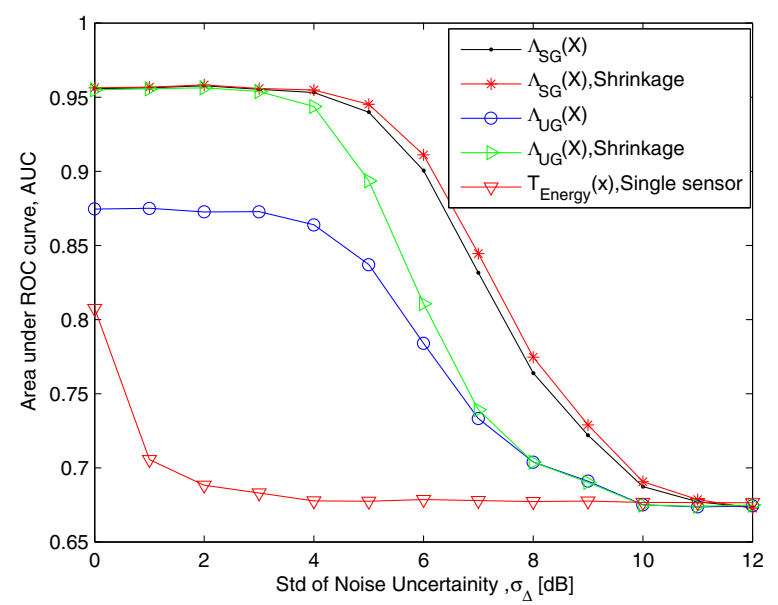

Figure 2 Area under the ROC curve (AUC) for analyzing the sensitivity to noise power uncertainty: $\sigma_{X}=0 \mathrm{~dB}, N=100, M=250, P_{0}=-7$ $\mathbf{d B}$ and $\sigma_{f}=\mathbf{0} \mathbf{d B}$. In this figure, we plot the area under the ROC curves (AUCs) to analyze the sensitivity to noise power uncertainty. Further details are given in subsection with the heading Experiment 2 in Section 6. 

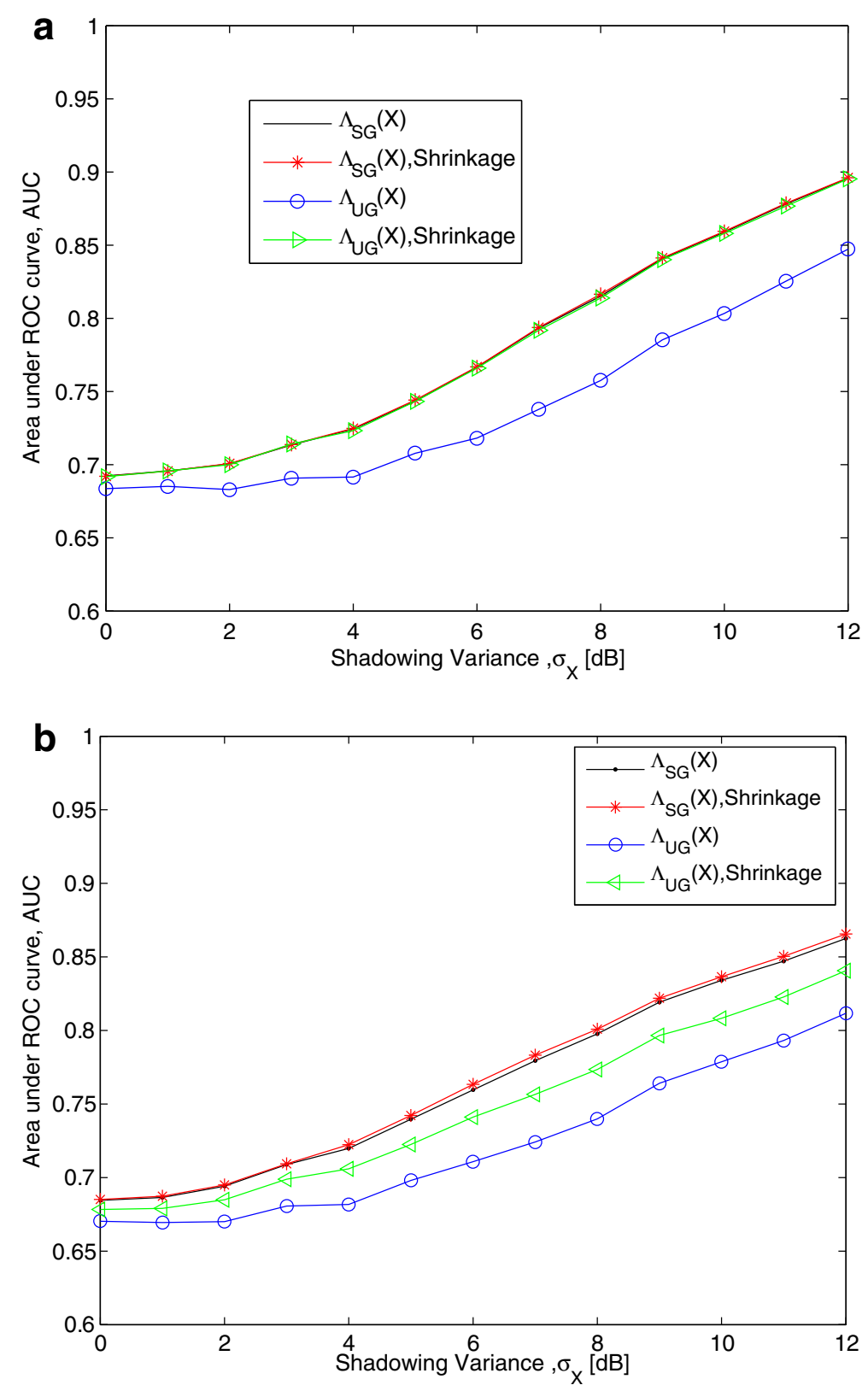

Figure 3 Area under the ROC curve (AUC) for analyzing the sensitivity to shadowing: $N=100$ and $M=250, P_{0}=-16 \mathrm{~dB}$ and $\sigma_{f}=\mathbf{0 d B}$. (a) ROC curves of $\sigma_{\Delta_{\sigma}}=0 \mathrm{~dB}$. (b) ROC curves of $\sigma_{\Delta_{\sigma}}=5 \mathrm{~dB}$. In this figure, we plot the area under the ROC curves (AUCs) to analyze the sensitivity to shadowing for the case when $P_{0}=-16 \mathrm{~dB}$ and $\sigma_{f}=0 \mathrm{~dB}$. Further details about this figure are given in subsection with the heading Experiment 3 in Section 6.

which helps to improve the overall performance for large $\sigma_{X}$ [41]. Similarly, in Figure 4, we plot the AUC curves for the high SNR regime. Figure $4 \mathrm{a}$ is for $\sigma_{\Delta_{\sigma}}=0 \mathrm{~dB}$, and Figure $4 \mathrm{~b}$ for $\sigma_{\Delta_{\sigma}}=5 \mathrm{~dB}$. Here again, we can see that using spatial signatures, the detection performance also improves compared to the unstructured GLRT $\Lambda_{\mathrm{UG}}(\mathbf{X})$, with or without shrinkage. Therefore, and particularly for large noise power uncertainty, it becomes clear again the superior performance of the structured
GLRT compared to any of the unstructured GLRT implementations.

\subsection{Experiment 4: Sensitivity to the available sample support for estimating the covariance matrix}

In this final experiment, we analyze the sensitivity to the observation interval length $N$, which has a direct impact on how accurate is the estimated covariance matrix and thus how reliable is the overall GLRT detection metric. 

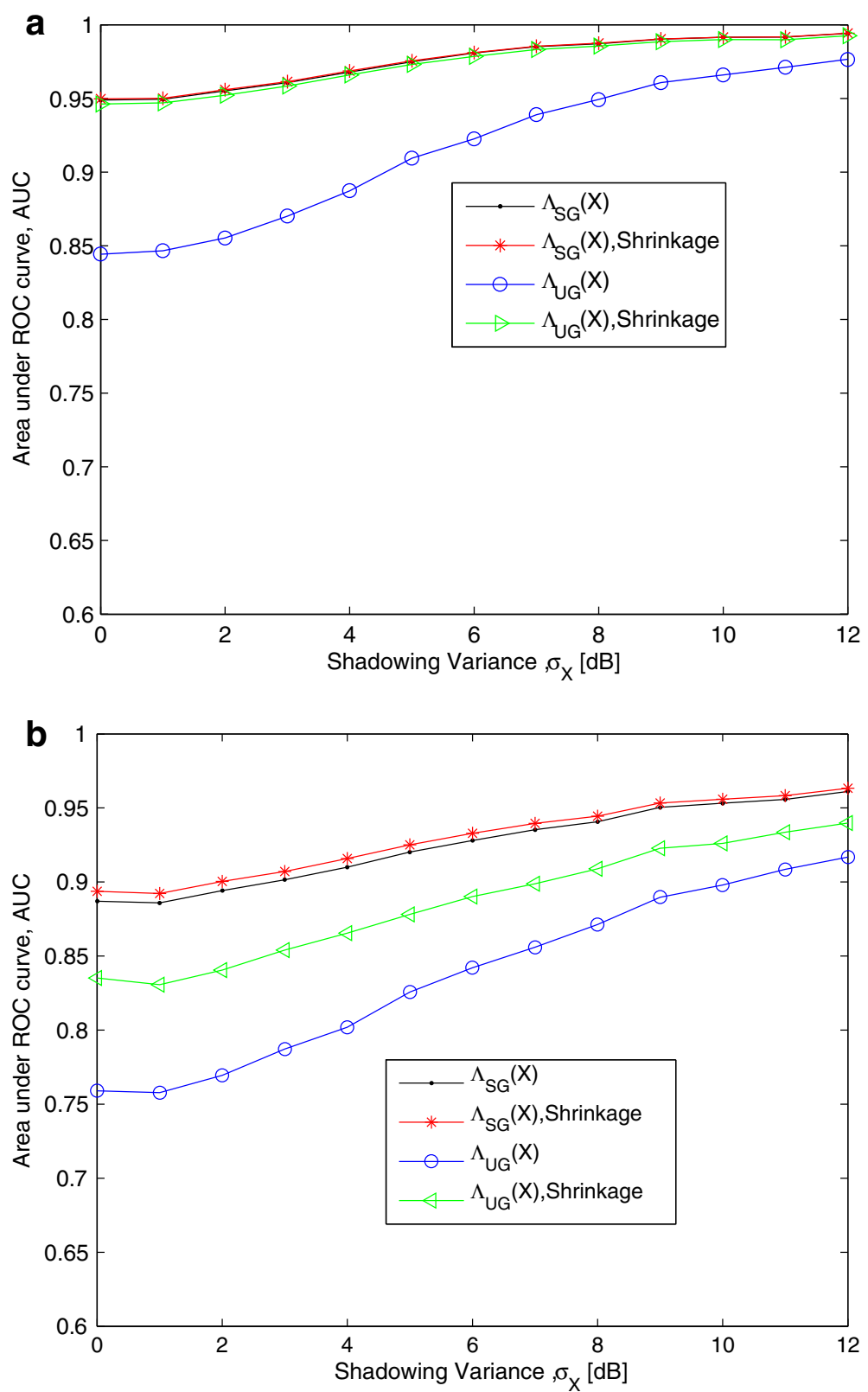

Figure 4 Area under the ROC curve (AUC) for analyzing the sensitivity to shadowing: $N=100$ and $M=\mathbf{2 5 0}, \boldsymbol{P}_{\mathbf{0}}=-\mathbf{7} \mathrm{dB}$ and $\sigma_{\mathrm{f}}=\mathbf{0} \mathrm{dB}$. (a) $\sigma_{\Delta_{\sigma}}=0 \mathrm{~dB}$ and (b) $\sigma_{\Delta_{\sigma}}=5 \mathrm{~dB}$. The figure shows the plots of the area under the ROC curves (AUCs) to analyze the sensitivity to shadowing for the case when $P_{0}=-7 \mathrm{~dB}$ and $\sigma_{f}=0 \mathrm{~dB}$. Further details about this figure are given in subsection with heading Experiment 3 in Section 6.

To do so, we consider again two different noise power uncertainties, $\sigma_{\Delta_{\sigma}}=\{0,5\} \mathrm{dB}$, as shown in Figure 5a,b, respectively. Taking a look at these figures, two important conclusions can be drawn. First, that the adoption of shrinkage becomes essential for very short observation intervals (i.e., when the number of measurements $N$ is smaller than the number of sensors $K$ ). In this case, the incorporation of shrinkage does actually prevent the illconditioning of the estimated covariance matrix, and this leads to a much higher AUC for both the unstructured and the structured GLRT. This can be observed in both Figure $5 \mathrm{a}$,b for $N<30$, since $K=30$ is the number of sensors being simulated in these experiments. The second conclusion is that for high noise power uncertainty (i.e., as in Figure $5 b$, the structured GLRT $\Lambda_{\mathrm{SG}}(\mathbf{X})$ clearly outperforms the unstructured GLRT $\Lambda_{\mathrm{UG}}(\mathbf{X})$. This is found to be true even for the case when the structured GLRT does not use shrinkage but the unstructured GLRT does, thus confirming the remarkable advantage of exploiting spatial information in uncertain scenarios. Moreover, the 

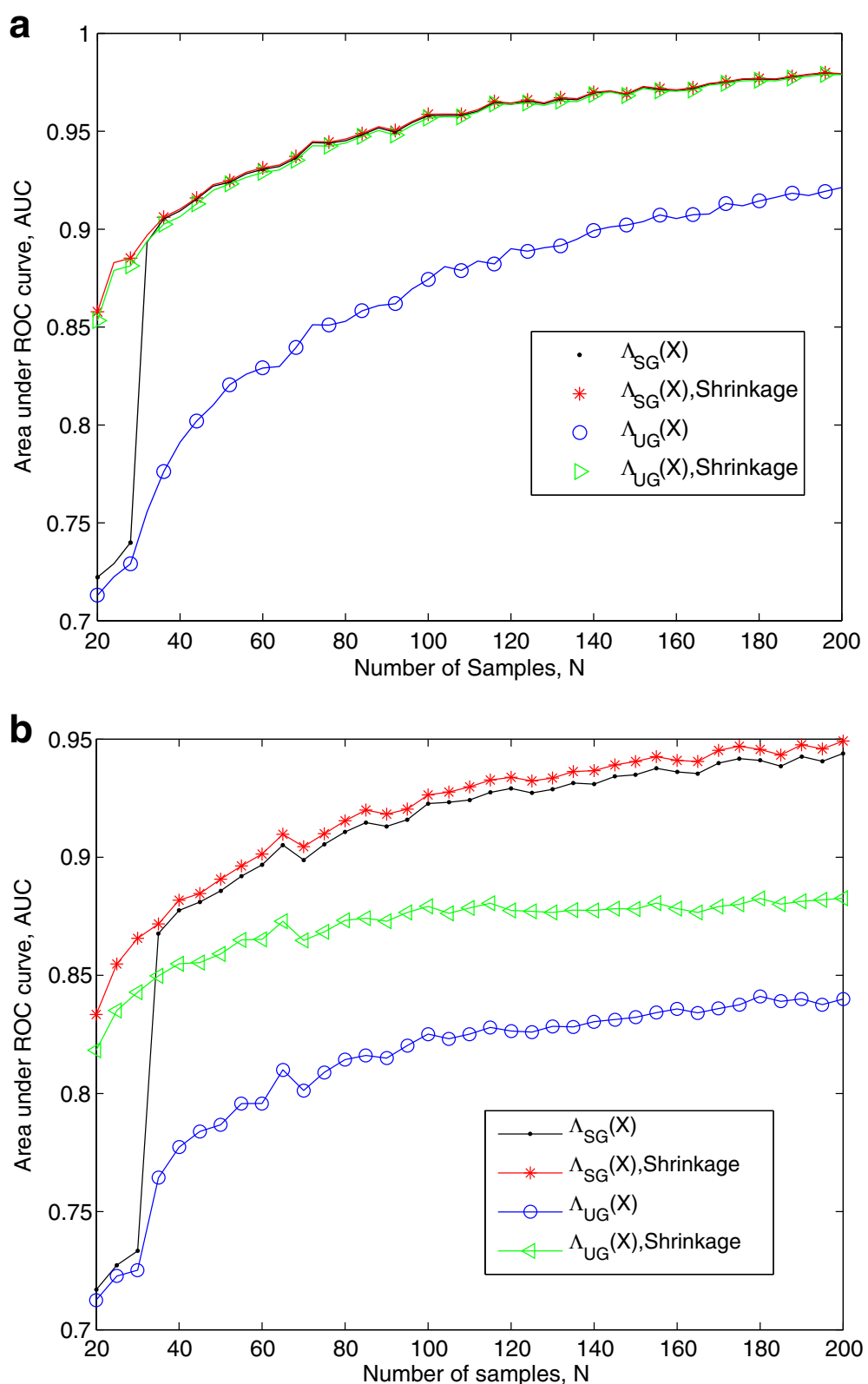

Figure 5 Area under the ROC curve (AUC) for analyzing the sensitivity to the observation interval length $N: \sigma_{\mathrm{X}}=\mathbf{3 \mathrm { dB }}, M=\mathbf{2 5 0}, P_{\mathbf{0}}=-\mathbf{7}$ $\mathbf{d B}$ and $\sigma_{f}=\mathbf{0} \mathbf{d B}$. (a) ROC curves of $\sigma_{\Delta_{\sigma}}=0 \mathrm{~dB}$. (b) ROC curves of $\sigma_{\Delta_{\sigma}}=5 \mathrm{~dB}$. The plots in this figure are of the area under the ROC curves (AUCs) to analyze the sensitivity to the observation length $N$. Details about this figure can be found in subsection with the heading Experiment 4 in Section 6 .

performance of the structured detector is not only better than the one provided by the unstructured one but also increases at a higher rate as a function of $N$. For that reason, we can also state that including spatial information results in a much more efficient exploitation of the information contained in the available measurements.

\section{Conclusion}

In this paper, a new GLRT-based collaborative spectrum sensing scheme has been proposed. The aim has been to achieve an improvement in the sensing performance by exploiting the implicit spatial correlation that is present among neighboring sensor nodes. Prior information on the sensor positions has been incorporated through a novel signal model based on the concept of spatial signatures, leading to the so-called structured GLRT detector, which is able to capture the correlation among different sensors. The performance of the proposed structured GLRT detector has been compared to that provided by the conventional unstructured GLRT by means of 
computer simulations. In order to further improve the detection performance, shrinkage estimation has been considered in both detectors as a way to circumvent the ill-conditioning problems that arise with short observation intervals. Interestingly, for the case of benign working conditions (i.e., in the absence of noise power uncertainty and shadowing), the use of shrinkage has been found to significantly improve the performance of the unstructured GLRT, leading to similar results to those provided by the use of spatial information in the structured GLRT. Nevertheless, this similar performance between both methods no longer holds when severe noise power uncertainty and shadowing do appear. In that case, the performance of the unstructured GLRT severely degrades, whereas the proposed structured GLRT is able to provide a more robust and superior performance. The results obtained for harsh working conditions confirm the suitability of this novel approach compared to traditional detectors that ignore spatial information.

\section{Endnote}

${ }^{\text {a}}$ For instance, in the case of IEEE 802.22 WRANs, sensors measure the entire $6 \mathrm{MHz}$ DTV channel at the Nyquist rate during observation intervals of $1 \mathrm{~ms}$, and thus, a total of $M=6 \cdot 10^{3}$ samples are typically processed per snapshot [26].

\section{Competing interests}

The authors declare that they have no competing interests.

\section{Acknowledgements}

This work was supported in part by the Spanish Ministry of Science and Innovation project TEC 2011-28219, by the Catalan Government under the grant FI-DGR-2011-FIB00711.

\section{Received: 6 December 2012 Accepted: 30 April 2013}

Published: 03 June 2013

\section{References}

1. S Haykin, Cognitive radio: Brain-empowered wireless communications. IEEE J. Select. Areas Commun. 23(2), 201-220 (2005)

2. KG Shin, H Kim, AW Min, A Kumar, Cognitive radios for dynamic spectrum access: from concept to reality. IEEE Wireless Commun. Mag. 17(6), 64-74 (2010)

3. Federal Communications Commission, Facilitating opportunities for flexible, efficient and reliable spectrum use employing cognitive radio technologies. (FCC 03-322, 2003) http://www.fcc.gov/document/facilitating-opportunities-flexibleefficient-and-reliable-spectrum-use-employing-cognitive

4. Y Zeng, YC Liang, Z Lei, SW Oh, F Chin, S Sun, in Proceedings of the IEEE Symposium on New Frontiers in Dynamic Spectrum (DySPAN). Worldwide regulatory and standardization activities on cognitive radio (Institute of Electrical and Electronics Engineers (IEEE), Singapore, 6-9 Apr 2010), pp. 1-9

5. R Tandra, A Sahai, SNR walls for signal detection. IEEE J. Select. Topics Signal Process. 2, 24-17 (2008)

6. AF Molisch, LJ Greenstein, M Shafi, Propagation issues for cognitive radio. Proc. IEEE. 97(5), 787-804 (2009)

7. TYucek, H Arslan, A survey of spectrum sensing algorithms for cognitive radio applications. IEEE Commun. Surveys Tutorials. 11, 116-130 (2009)

8. Z Quan, S Cui, HV Poor, AH Sayed, Collaborative wideband sensing for cognitive radios. IEEE Signal Processing Mag. 25(6), 60-73 (2008)
9. IF Akyildiz, MC Vuran, OB Akan, in Proceedings of the International Symposium on Modeling and Optimization in Mobile, Ad Hoc, and Wireless Networks (WiOpt). On exploiting spatial and temporal correlation in wireless sensor networks (Institute of Electrical and Electronics Engineers (IEEE), Paderborn, 14-18 May 2004), pp. 71-80

10. A Ghasemi, ES Souca, Asymptotic performance of collaborative spectrum sensing under correlated log-normal shadowing. IEEE Commun. Lett. 11, 34-36 (2007)

11. B Kasiri, C Jun, in Proceedings of the IEEE Conference on Computer Communications (INFOCOM). Effects of correlated shadowing on soft decision fusion in cooperative spectrum sensing (Institute of Electrical and Electronics Engineers (IEEE) San Diego, 15-19 Mar2010), pp. 1-6

12. TW Anderson, An Introduction to Multivariate Statistical Analysis. (Wiley, Hoboken, 2003), pp. 376-446

13. Y Zeng, YC Liang, Spectrum-sensing algorithms for cognitive radio based on statistical covariances. IEEE Trans. Veh. Technol. 58(4), 1804-1815 (2009)

14. Y Zeng, YC Liang, Eigenvalue-based spectrum sensing algorithms for cognitive radio. IEEE Trans. Commun. 57(6), 1784-1793 (2009)

15. H Celebi, H Arslan, Utilization of location information in cognitive wireless networks. IEEE Wireless Commun. Mag.

14(4), 6-13 (2007)

16. P Jia, M Vu, T Le-Ngoc, in Proceedings of the Asilomar Conference on Signals, Systems and Computers. Location-aware cognitive sensing for maximizing network capacity (Institute of Electrical and Electronics Engineers (IEEE), Pacific Grove, 1-4 Nov 2009), pp. 78-82

17. T Zue, Y Shi, X Dong, A framework for location-aware strategies in cognitive radio systems. 1, 30-33 (2012)

18. E Visotsky, S Kuffner, R Peterson, in Proceedings of the IEEE Symposium on New Frontiers in Dynamic Spectrum (DySPAN). On collaborative detection of TV transmissions in support of dynamic spectrum sharing (Institute of Electrical and Electronics Engineers (IEEE), Baltimore, 8-11 Nov 2005), pp. 338-345

19. D Astely, A Swindlehurst, B Ottersten, Spatial signature estimation for uniform linear arrays with unknown receiver gains and phases. IEEE Trans. Signal Process. 47(8), 2128-2138 (1999)

20. S Ali, JA López-Salcedo, G Seco-Granados, in Proceedings of the European Conference on Signal Processing (EUSIPCO). Improved GLRT based on the exploitation of spatial correlation between neighbouring sensors (Proceedings of the European Conference on Signal Processing, Barcelona, 27 Aug 2011), pp. 1045-1049

21. J Schafer, K Strimmer, A shrinkage approach to large-scale covariance matrix estimation and implications for functional genomics. Stat. Appl. Genet. Mol. Biol. 4, 1-30 (2005)

22. SJ Shellhammer, S Shankar, R Tandra, J Tomcik, in Proceedings of the International Workshop on Technology and Policy for Accessing Spectrum (TAPAS). Performance of power detector sensors of DTV signals in IEEE 802.22 WRANs (ACM, Boston, 5 Aug 2006), pp. 4-13. http://dl.acm.org/citation.cfm?id=1234392

23. R Niu, PK Varshney, Distributed detection and fusion in a large wireless sensor network of random size. EURASIP J. Wireless Commun. Netw. 4 462-472 (2005)

24. YR Tsai, Sensing coverage for randomly distributed wireless sensor networks in shadowed environments. IEEE Trans. Veh. Technol. 57, 556-564 (2008)

25. P Belanovic, S Valcárcel-Macua, S Zazo, in Proceedings of the IEEE International Conference on Acoustics, Speech and Signal Processing (ICASSP). Location-aided distributed primary user identification in a cognitive radio scenario (Institute of Electrical and Electronics Engineers (IEEE), Kyoto, 25-30 Mar 2012), pp. 1-4

26. AW Min, KG Shin, X Hu, Secure cooperative sensing in IEEE 802.22 WRANs using shadow fading correlation. IEEE Trans. on Mobile Comput. 10(10), 1434-1447 (2011)

27. PK Varshney, CS Burrus, Distributed detection and data fusion. (Springer New York, 1997)

28. S Shellhammer, G Chouinard, Spectrum sensing requirements summary. IEEE P802.22-06/0089r1, Tech. Rep. (2006)

29. M Di Renzo, L Imbriglio, F Graziosi, F Santucci, in Proceedings of the IEEE Global Telecommunications Conference (GLOBECOM). Cooperative spectrum sensing over correlated log-normal sensing and reporting 
channels (Institute of Electrical and Electronics Engineers (IEEE), Honolulu, 30 Nov-4 Dec 2009), pp. 1-8

30. P Stoica, Y Selen, Model-order selection: a review of information criterion rules. IEEE Signal Processing, Mag. 21(4), 36-47 (2004)

31. LL Scharf, Statistical signal processing. Detection, estimation and time series analysis. (Addison-Wesley, New York, 1991), pp. 379-413

32. SM Kay, Fundamentals of statistical signal processing. Detection theory, vol. 2. (Prentice Hall, Upper Saddle River, 1998)

33. CXu, SM Kay, in Proceedings of the IEEE Radar Conference. On centralized composite detection with distributed sensors (Institute of Electrical and Electronics Engineers (IEEE), Rome, 26-30 May 2008), pp. 1-6

34. G Vázquez-Vilar, D Ramírez, R López-Valcarce, J Vía, I Santamaría, in Proceedings of the International Conference on Cognitive Radio and Advanced Spectrum Management (CogART). Spatial rank estimation in cognitive radio networks with uncalibrated multiple antennas (ACM, New york Barcelona, 26-29 Oct 2011), pp. 1-5

35. D Ramírez, G Vázquez-Vilar, R López-Valcarce, JVía, Santamaría I, Detection of rank-P signals in cognitive radio networks with uncalibrated multiple antennas. IEEE Trans. Signal Process. 59, 3764-3774 (2011)

36. HLV Trees, Detection, estimation, and modulation theory. Optimum array processing, vol. 4. (Wiley, New York, 2002), pp. 984-1018

37. C Stein, Inadmissibility of the usual estimator of the mean of a multivariate normal distribution. Proc. Berkeley Symposium on Mathematics, Statistiscs and Probability. 1, 197-206 (1956)

38. Y Chen, A Wiesel, AO Hero, in Proceedings of the IEEE International Conference on Acoustics, Speech and Signal Processing (ICASSP). Shrinkage estimation of high dimensional covariance matrices (Institute of Electrical and Electronics Engineers (IEEE), Taipei, 19-24 Apr 2009), pp. 2937-2940

39. W Lin, Q Zhang, in Proceedings of the IEEE International Conference on Communication Systems. A design of energy detector in cognitive radio under noise uncertainty (Institute of Electrical and Electronics Engineers (IEEE), Guangzhou, 19-21 Nov 2008), pp. 213-217

40. T Fawcett, in Tech. Rep. HPL-2003-4. ROC graphs: notes and practical considerations for researchers (HP Laboratories Palo Alto, 2003), pp. 1-20

41. T Muetze, P Stuedi, F Kuhn, G Alonso, in Proceedings of the IEEE Conference on Sensor, Mesh and Ad Hoc Communications and Networks (SECON). Understanding radio irregularity in wireless networks (Institute of Electrical and Electronics Engineers (IEEE), San Francisco, 16-20 Jun 2008), pp. 82-90

doi:10.1186/1687-1499-2013-150

Cite this article as: Ali et al:: Spectrum sensing with spatial signatures in the presence of noise uncertainty and shadowing. EURASIP Journal on Wireless Communications and Networking 2013 2013:150.

\section{Submit your manuscript to a SpringerOpen ${ }^{\mathcal{O}}$ journal and benefit from:}

- Convenient online submission

- Rigorous peer review

- Immediate publication on acceptance

- Open access: articles freely available online

- High visibility within the field

- Retaining the copyright to your article

Submit your next manuscript at $\boldsymbol{\wedge}$ springeropen.com 